EGU2020-12036, updated on 08 May 2020

https://doi.org/10.5194/egusphere-egu2020-12036

EGU General Assembly 2020

(c) Author(s) 2020. This work is distributed under

the Creative Commons Attribution 4.0 License.

\title{
A transatlantic, multidisciplinary graduate school focussed on the North Atlantic Ocean: rationale, challenges, lessons-learned, achievements and benefits.
}

Douglas Wallace ${ }^{1}$, Markus Kienast ${ }^{1}$, Kirsten Laing ${ }^{1}$, Brendal Townsend ${ }^{1}$, Christian Dullo ${ }^{2}$, Colin Devey $^{2}$, Christel van den Bogaard ${ }^{2}$, and Tatiana Cabral ${ }^{3}$

${ }^{1}$ Oceanography Dept., Dalhousie University, Halifax, Nova Scotia, Canada

${ }^{2}$ Helmholtz Centre for Ocean Research Kiel (GEOMAR), Kiel, Germany

${ }^{3}$ Fazenda de Camarao, Calhau, Sao Vicente, Cabo Verde

Increasingly, careers in ocean research are international and lie outside traditional employment sectors (academia and government). In response to a need for training that prepares the next generation of ocean scientists for a globalized, multisectoral environment, we initiated a transatlantic, multi-disciplinary graduate school which connected students and their supervisors in Halifax, Canada and Kiel, Germany. We took advantage of complementary capacities and cultures on both sides of the Atlantic to create a training program that conveyed technical and research skills in ocean science and advanced technology, and promoted the ability to manage deep sea and open ocean environments. Our goal was to provide each graduate with an international network and the ability to work effectively as an "advocate for the ocean".

The transatlantic graduate school was supported from 2012 to 2020 with funding obtained, separately, from the Natural Sciences and Engineering Research Council's CREATE program in Canada and the Helmholtz Association's Graduate Research School program in Germany. The NSERC CREATE Transatlantic Ocean System Science and Technology (TOSST) was based at Dalhousie University, whereas the Helmholtz Ocean System Science and Technology (HOSST) graduate school was based at the Helmholtz Centre for Ocean Research Kiel (GEOMAR) with participation from the Christian-Albrecht University of Kiel. TOSST supported a total of $20 \mathrm{PhD}$ and 3 Masters candidates. HOSST supported 24 doctoral researchers. The participants were recruited into 2 cohorts.

The participants' disciplines ranged from marine geology to atmospheric physics and included molecular ecology, marine conservation as well as social and policy sciences, etc.. The common focus was on the Atlantic Ocean and on value-added training that addressed business skills as well as economic, regulatory, management and cultural aspects relevant to Atlantic Ocean spaces. A program of annual Summer Schools included two held in a small island developing state (Cabo Verde), with participation of African students.

TOSST-HOSST did not attempt to unify the disparate academic systems of Germany and Canada 
but, instead, focussed on connecting and broadening the experience of young researchers. The program sought to maximise the value of transatlantic scientific cooperation, convey broad experience and skills beyond students' individual projects and disciplines, and create a diverse community of scholars who could work effectively together.

The presentation will highlight benefits and challenges encountered, on both sides of the Atlantic, and lessons learned during the program. Examples of lessons learned included: the value of a bilateral, cohort model for building networks of young researchers over long distances (as opposed to more distributed, multi-institutional networks); the importance of regular (effective) videoconferencing as well as (occasional) face-to-face meetings; the importance of program coordinators for overcoming barriers to international exchanges; the risk of overburdening the participants with program requirements without compensation of the academic requirements of home institutions; the need for supervisors to commit to the international aspects of the program; the value of exposure to radically different research environments (including those in developing countries). 